\title{
Artificial Neural Network System in the Automated Diagnosis of Fetal Heart Rate
}

\author{
$\mathrm{K} \mathrm{Maeda}^{1 *}, \mathrm{Y}$ Noguchi $^{2}$, F Matsumoto ${ }^{2}$ and T Nagasawa ${ }^{3}$
}

${ }^{1}$ Department of Obstetrics and Gynecology (emeritus) of Tottori University Medical School, Yonago, Japan

${ }^{2}$ Department of Applied Physics, National Defense Academy, Yokosuka, Japan

${ }^{3}$ Department of Information Technology, TOITU Ltd, Tokyo, Japan

\begin{abstract}
Fetal heart rate (FHR) tracing with uterine contraction is the most commonly utilized for fetal monitoring during pregnancy and in the labor. Automated computerized diagnosis of FHR was intended and recently introduced artificial neural network analysis because of its very objective nature. FHR tracing was quantified and FHR score was obtained, FHR frequency power spectrum was analyzed to diagnose sinusoidal FHR to apply in artificial neural network analysis. The neural network was composed of a soft ware with three layers associated with back-propagation system, and it was trained with 8 FHR parameters including the sinusoidal FHR for 10,000 times to obtain $100 \%$ correct internal check. Trained network soft ware was copied with new computer to diagnose new subjects. Diagnostic input was the $8 \mathrm{FHR}$ parameters of 3 periods of $5 \mathrm{~min}$, and output was probabilities to be normal, intermediate and pathologic outcomes in percentage, of which diagnosis was correct in the comparison to simultaneously calculated FHR score that was high in pathologic outcome probability, moderate in intermediate and low in normal outcome probability. Neural network index derived from pathologic and normal outcome probabilities was useful in the outcome prediction of prolonged fetal monitoring.
\end{abstract}

Keywords: Fetus; Fetal Heart Rate; Automatic Diagnosis; Neural Network Analysis; Software; FHR Score; Outcome Probability

\section{Introduction}

Although continuous fetal heart rate (FHR) pattern monitoring has been improved fetal outcome [1-3] with visual diagnosis of anteand intra-partum cardiotocogram (CTG), it was time consuming and confused by inter-observer difference and false positive FHR changes. Among various fetal evaluations investigated with computerized CTG analysis, we intended to objectively study quantitative FHR changes with automated analysis. Continuous FHR curve was obtained by ultrasonic Doppler autocorrelation FHR meter, and uterine contraction was traced with external tocometry. Each 150 data of FHR and uterine contraction were sampled in every 5 min continuously and analyzed by three programs including an experts' knowledge system, the FHR baseline frequency power spectrum analyzer and artificial neural network soft ware composed of 3 layers and back-propagation system analyzing 8 FHR parameters to achieve probabilities to be pathologic, intermediate and normal outcome.

\section{Methods}

\section{Quantification of FHR to determine FHR score in the experts' knowledge system}

FHR changes were quantified by the baseline FHR, long term variability, acceleration and deceleration (duration, amplitude, nadir heart rate, recovery time and lag time) in every 5 minutes (Figure 1).

FHR baseline was determined by averaging the FHR data found in the step of the most frequent FHR data among $10 \mathrm{bpm}$ steps of FHR. FHR accelerations were detected by transient FHR rise above the baseline for 15 or more beats per minute (bpm) and 15 or more seconds' duration after 30 weeks of pregnancy. FHR decelerations were determined by the 15 or more sec duration and $15 \mathrm{bpm}$ or more decrease to the baseline. Decelerations were analyzed by the DIP SHAPE value (dip area/(deceleration amplitude $\mathrm{x}$ duration)), intra- deceleration variability that was the sum of every heart rate difference between adjacent FHR data in the deceleration, and lag time between the contraction peak and the deceleration nadir. Late deceleration was defined if the DIP SHAPE was less than 0.5 and intra-deceleration variability was smaller than 60 , lag time was 20 or more sec and its number was almost the same as uterine contraction in $15 \mathrm{~min}$. DIP SHAPE was $<0.5$ and lag time was almost zero in the early deceleration, and DIP SHAPE was larger than 0.6 and intra-deceleration variability was larger than 60 in variable deceleration in the automated FHR analysis. Duration was longer than $1 \mathrm{~min}$ and nadir heart rate was $<100$

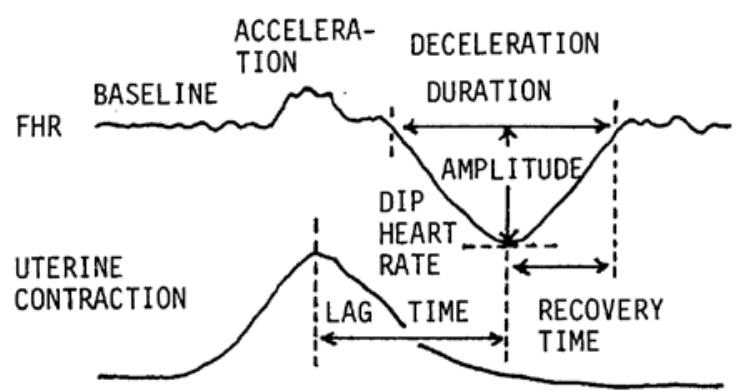

Figure 1: Quantified analysis of FHR changes.

*Corresponding author: Kazuo Maeda, Department of Obstetrics and Gynecology, Tottori University Medical School, 3-125, Nadamachi, Yonago, Tottoriken, 6830835 Japan, Tel: 81-859-22-6856; E-mail: maedak@mocha.ocn.ne.jp

Received December 08, 2011; Accepted January 05, 2012; Published January 14,2012

Citation: Maeda K, Noguchi Y, Matsumoto F, Nagasawa T (2012) Artificial Neural Network System in the Automated Diagnosis of Fetal Heart Rate. J Health Med Informat S5. doi:10.4172/2157-7420.S5-001

Copyright: (C) 2012 Maeda K, et al. This is an open-access article distributed under the terms of the Creative Commons Attribution License, which permits unrestricted use, distribution, and reproduction in any medium, provided the original author and source are credited. 
$\mathrm{bpm}$ in the severe variable deceleration. The duration was $>2 \mathrm{~min}$ in prolonged duration.

Evaluation scores were determined in each FHR changes by the incidence of neonatal depression in the FHR change assessed by the Apgar score lower than 7, and the sum of evaluation scores in 5 minutes formed FHR score (Figure 1 and Table 1), where the fetus was abnormal if the FHR score was 10 or more and highly abnormal if the FHR score was $>20$ [4]. FHR baseline long term variability (LTV) was determined by the mean of downhill amplitudes of FHR data [5].

\section{FHR baseline frequency power spectrum}

The spectrum was obtained by the fast Fourier transform (FFT) frequency analysis, to diagnose sinusoidal FHR, i.e. the area under low frequency spectrum $(0.03125-0.1 \mathrm{~Hz})$ to the area under total spectrum $(\mathrm{La} / \mathrm{Ta})$ and peak power spectrum density (PPSD) were determined to diagnose ominous sinusoidal FHR and differentiate physiologic benign sinusoidal FHR [6].

\section{Artificial neural network system}

Each of 8 FHR parameters was used in the three succeeding 5 minutes that was an $8 \times 3$ pattern served as an input. Twenty cases with a known outcome were selected as teaching patterns for supervised leaning: 3 normal, 3 Intermediate and 14 pathologic cases, but not programmed by the experts' knowledge. Although it is ideal to train the neural network system with full FHR data of known outcome cases, our system was a hybrid neural network utilizing processed FHR parameters in the preceding analyzing computer, and sinusoidal FHR diagnosed by frequency power spectrum, to quickly analyze the data in the intrapartum monitoring. The output signals designated to the probability of a normal, intermediate and pathological outcome. Then $\mathrm{O} 1, \mathrm{O} 2$, and $\mathrm{O} 3$ were taken as an output vector where $\mathrm{O} 1$ indicates normal, $\mathrm{O} 2$ intermediate and $\mathrm{O} 3$ pathological. The output vector should be $(1,0,0),(0,1,0)$ and $(0,0,1)$, if the input pattern is normal, intermediate and pathological, respectively. We designed the neural network as 3 layers: 24 units for input layer, 30 units for hidden (intermediate) layer, and 3 units for output layer. The output value means certainty, and to obtain probability, the output values are divided by the sum of three output values, and then the probability was clear, i.e. The probabilities to be normal outcome, those of intermediate and pathological outcome were shown in the output data.

Trained neural network software was installed into other computers to diagnose new cases. Eight FHR parameters, which were the same as used in the training of artificial neural network in three 5 minutes'

\begin{tabular}{|l|l|l|}
\hline PARAMETER & ABNORMAL SIGN & EVALUATION SCORE \\
\hline BASELINE FHR & $110-130$ or $160-180$ & 1 \\
\hline & $>110 \mathrm{bpm}$ or $>180 \mathrm{bpm}$ & 3 \\
\hline DECELERATION & & \\
\hline Duration & $>60 \mathrm{sec}$ & 3 \\
\hline Amplitude & $>50 \mathrm{bpm}$ & 2 \\
\hline Dip heart rate & $<100 \mathrm{bpm}$ & 2 \\
\hline Recovery time & $>40 \mathrm{sec}$ & 3 \\
\hline Lag time & $>40$ sec & 3 \\
\hline & No accompanied acceleration & 2 \\
\hline & W-shaped deceleration & 4 \\
\hline
\end{tabular}

FHR SCORE = sum of evaluation scores in $5 \mathrm{~min}$

Table 1: Calculation of FHR score. periods were input into trained neural network and its outputs were three probabilities to be normal, intermediate and pathologic outcome expressed by the percentage which was easily understood without education [7].

The neural index was the mean of past normal outcome probability subtracted by the mean of past pathologic outcome probability determined in every 5 minutes [8].

\section{Results}

\section{FHR score}

Maximal FHR score in the $1^{\text {st }}$ stage of labor correlated with Apgar score, i.e. Apgar score $=10.04-0.34$ (Maximal FHR score in the $1^{\text {st }}$ stage of labor), $\mathrm{R}^{2}=0.69, \mathrm{p}<0.001, \mathrm{~N}=18$, where the Apgar score was 6 and the neonate was expected to be mild asphyxia if the FHR score was 10 , while Apgar score was 3 , where severe asphyxia was expected, if maximal FHR score was 20 [9]. Therefore, interventional delivery was indicated if the FHR score was high in early stage of labor.

Also the maximal FHR score in the $1^{\text {st }}$ stage of labor correlated with umbilical arterial blood $\mathrm{pH}$ and base excess, i.e. Umbilical arterial $\mathrm{BE}=-(0.63 \mathrm{Max}$ FHR score +4.02$), \mathrm{R}^{2}=0.86, \mathrm{p}=0.01, \mathrm{~N}=5$. The $2^{\text {nd }}$ stage FHR score correlated fetal scalp blood $\mathrm{pH}$ [4]. In addition, the fetal distress index (FDI), which was derived from FHR score, loss of variability, late deceleration etc, was 3 or more 30 min before delivery, when the umbilical cord blood was acidosis [10], i.e. fetal acidosis may be estimated by detailed FHR analysis.

\section{Frequency power spectrum}

It was ominous sinusoidal FHR when the $\mathrm{La} / \mathrm{Ta}$ ratio was $>=39 \%$ and at the same time PPSD was $>=300 \mathrm{bpm}^{2} / \mathrm{Hz}$. Physiological benign sinusoidal FHR was diagnosed if the La/Ta ratio was lower than $39 \%$ or PPSD was lower than $300 \mathrm{bpm}^{2} / \mathrm{Hz}$ and differentiated from ominous sinusoidal FHR [6]. Ominous sinusoidal FHR was one of neural network training and diagnostic parameters. Also severe loss of FHR baseline variability was diagnosed if $\mathrm{La} / \mathrm{Ta}$ ratio was $<15 \%$ and PPSD was $<60 \mathrm{bpm}^{2} / \mathrm{Hz}[11]$.

\section{Artificial neural network}

Performance of the neural network computer: The recognition results obtained by the neural network with teaching input after training for 10,000 times, all data carried the probabilities of 0.998 (or $99.8 \%$ ) to1.000 (or $100 \%$ ) (Table 2). Therefore, this well-trained neural network can be used to recognize new patients not included in the teaching patterns that is to say "open recognition" [12].

Neural network computer results and neonatal state: The normal and pathological outcome probabilities obtained by the neural network were compared to the neonatal states evaluated by the Apgar score, clinical condition and umbilical cord blood $\mathrm{pH}$, where analysis time was as long as 50 minutes and the neural network training data was also obtained in 50 minutes. The neonatal states matched outcome probabilities in 25 out of 29 cases ( $86 \%)$.

The accuracy of neural network computer diagnosis was confirmed by the comparison to simultaneously determined FHR scores, which were high in the pathologic outcome probability, moderate in intermediate outcome probability, and low in normal outcome probability [4], i.e. mean \pm SD was:

FHR scores in pathologic outcome probability $=18.60 \pm 6.00(\mathrm{~N}=5)$ 
Citation: Maeda K, Noguchi Y, Matsumoto F, Nagasawa T (2012) Artificial Neural Network System in the Automated Diagnosis of Fetal Heart Rate. J Health Med Informat S5. doi:10.4172/2157-7420.S5-001

Page 3 of 4

\begin{tabular}{|c|c|c|c|c|}
\hline \multirow[t]{2}{*}{ Case No. } & \multirow[t]{2}{*}{ FHR } & \multicolumn{3}{|c|}{ Probability } \\
\hline & & Normal & Intermediate & Pathological \\
\hline 1 & SVD & 0 & 0.001 & 0.999 \\
\hline 2 & VD & 0.001 & 0.999 & 0 \\
\hline 3 & normal & 0.998 & 0.001 & 0.001 \\
\hline 4 & normal & 0.998 & 0.001 & 0.001 \\
\hline 5 & sinusoidal & 0 & 0 & 1 \\
\hline 6 & bradycardia & 0.001 & 0 & 0.999 \\
\hline 7 & SVD & 0 & 0.001 & 0.999 \\
\hline 8 & LD & 0 & 0 & 1 \\
\hline 9 & LV & 0 & 0.001 & 0.999 \\
\hline 10 & normal & 0.998 & 0.001 & 0.001 \\
\hline 11 & VD & 0.001 & 0.888 & 0.001 \\
\hline 12 & VD & 0.001 & 0.998 & 0.001 \\
\hline 13 & LV & 0 & 0 & 1 \\
\hline 14 & LV & 0 & 0 & 1 \\
\hline 15 & LD & 0 & 0 & 1 \\
\hline 16 & LD & 0 & 0 & 1 \\
\hline 17 & bradycardia & 0.001 & 0 & 0.999 \\
\hline 18 & bradycardia & 0.001 & 0 & 0.999 \\
\hline 19 & sinusoidal & 0 & 0 & 1 \\
\hline 20 & SVD & 0 & 0.001 & 0.999 \\
\hline
\end{tabular}

SVD: severe variable deceleration

VD: variable deceleration

LD: late deceleration

LV: loss of variability

Table 2: Recognition probability of 20 cases used in the network training.

Those in intermediate outcome probability $=13.35 \pm 3.65(\mathrm{~N}=15)$

Those in normal outcome probability $=1.33 \pm 1.41(\mathrm{~N}=9)$

Significant differences were noted among each group.

Therefore, the outcome probabilities obtained by the artificial neural network will correctly indicate fetal outcome. Furthermore, fetal outcome was normal when final neural index was $>0$, while the neonatal Apgar score and umbilical blood $\mathrm{pH}$ were low in the cases whose neural index was $<0$. The index was suitable to predict the outcome in hardly diagnostic prolonged fetal monitoring [5].

\section{Discussion}

The software of artificial neural network was trained with the FHR parameters of known outcome cases to achieve objective diagnosis by the probability to be normal, intermediate and pathological outcome in our study. The problem of unstable inter-observer difference would be solved after introduction of the network diagnosis. The construction and education of neural network in the soft ware will guarantee the uniform diagnostic results by the implantation of educated network software copy into the other computer. It will be the advantage of software network to the use of software block which would be educated in every network computer.

The sinusoidal FHR was one of FHR parameters which were used in the training of the neural network. The sinusoidal FHR was difficult to diagnose in the past but we found that physiological benign sinusoidal FHR was differentiated from ominous one by the association of periodic fetal respiration recorded by actocradiogram [13]. As it was difficult to include the method to automated FHR diagnosis which was active only by the FHR, we analyzed frequency power spectrum of FHR and achieved the sinusoidal FHR diagnosis by using heart rate material
[6] and applied the method to the training and diagnosis of the neural network.

Since the importance of each FHR pattern has been emphasized in the common visual FHR pattern classification; e.g. late deceleration, loss of variability, prolonged deceleration, non-reactive FHR pattern, bradycardia, tachycardia, doctors may be confused by the massive information of independent signs. Hence it will be recommended now more comprehensively to inform fetal status. Therefore the probability of fetal outcome estimated by the neural network and the FHR score of experts' knowledge system, which comprehensively evaluate the fetal status, will inform the fetal status from the composite view point. For example in our central-computerized fetal monitoring, abnormality of each FHR parameter and at the same time FHR score and outcome probability are announced attending doctor to make it possible to evaluate comprehensive fetal condition.

\section{Conclusion}

Automated computerized FHR diagnosis including artificial neural network system will save the time and hard work of obstetric staffs from continuous watching FHR tracing in the labor with the accurate FHR diagnosis and prompt information of FHR abnormality. Multiple monitoring is also possible with the computer. Artificial neural network analysis contributes perinatal medicine not only by correct FHR diagnosis but also by the reduced inter-observer difference.

\section{References}

1. Maeda K (1979) Healthy baby and reduced fetal death by improved obstetric management. In Karim SMM Yan Ki (eds) Problems in Perinatal Medicine. Proc $1^{\text {st }}$ Asia Oceania Perinatal Medicine, Singapore University 525-529.

2. Takeshita K, Ando Y, Ohtani K, Takashima S (1989) Cerebral palsy in Tottori, Japan. Benefits and risks of progress in perinatal medicine. Neuroepidemiology 4: 184-192. 
Citation: Maeda K, Noguchi Y, Matsumoto F, Nagasawa T (2012) Artificial Neural Network System in the Automated Diagnosis of Fetal Heart Rate. J Health Med Informat S5. doi:10.4172/2157-7420.S5-001

3. Tsuzaki T, Sekijima A, Morishita K, Takeuchi Y, Mizuta M, et al. (1990) The survey on the perinatal variables and the incidence of cerebral palsy for 12 years before and after the application of the fetal monitoring system. Nippon Sanka Fujinka Gakkai Zasshi 42: 99-105.

4. Maeda K, Kimura S, Fukui Y, Ozawa S, Kosaka W, et al. (1969) Pathophysiology of Fetus. Fuikuoka Printing, Fukuoka.

5. Maeda K, Arima T, Tatsumura M, Nagasawa T (1980) Computer-aided fetal heart rate analysis and automatic fetal-distress diagnosis duting labor and pregnancy utilizing external technique in fetal monitoring. In DAB Lindburg, S Kaihara (eds) MEDINFO 80, North Holland, Amsterdam: 1214-1218.

6. Maeda K, Nagasawa T (2005) Automatic computerized diagnosis of fetal sinusoidal heart rate. Fetal Diag Ther 20: 328-334.

7. Maeda K, Utsu M, Makio A, Serizawa M, Noguchi Y, et al. (1998) Neura network analysis of fetal heart rate. J Matern-Fetal Investig 8: 163-171.

8. Maeda K, Noguchi Y, Matsumoto F (2003) Evaluation of prolonged feta monitoring with normal and pathologic outcome probabilities determined by artificial neural network. Fet Diag Ther 18: 370-375.

9. Maeda K, Noguchi Y, Matsumoto F, Nagasawa T (2006) Quantitative fetal heart rate evaluation without pattern classification: FHR score and artificial neura network analysis. In A Kurjak, FA Chervenak (eds) Text book or Perinatal Medicine, Informa, UK 1487-1495.

10. Irie T (1986) Automated fetal heart rate analysis and its trendgram in relation to the gas analysis and acid base balance of umbilical cord arterial blood. Nippon Sanka Fujinka Gakkai Zasshi 38: 1623-1631.

11. Maeda K, Nagasawa T (2010) Loss of FHR variability diagnosed by frequency analysis. J Perinat Med 38: 197-201.

12. Yasuaki Noguchi, Fujihiko Matsumoto, Kazuo Maeda and Takashi Nagasawa (2009) Neural network analysis and evaluation of fetal heart rate. Algorithms 2: $19-30$.

13. Ito T, Maeda K, Takahashi H, Nagata N, Nakajima K, et al. (1994) Differentiation between physiologic and pathologic sinusoidal FHR pattern by fetal actocardiogram. J Perinat Med 22: 39-43.
This article was originally published in a special issue, Aid of Intelligent Systems: Computers handled by Editor(s). Dr. Stephen Obol Opiyo, Ohio State University, United States 